\title{
50 IN-SITU VISUALIZATION AND MEASUREMENT OF TUMOR-INFILTRATING LYMPHOCYTES (TILS) ON INTACT FFPE RENAL CELL CARCINOMA (RCC) TISSUE USING THE SPATIAL MOLECULAR IMAGER (SMI)
}

${ }^{1}$ Evan Newell, ${ }^{2}$ Youngmi Kim*, ${ }^{1}$ Heeju Ryu, ${ }^{1}$ Shamin Li, ${ }^{2}$ Michael Leon, ${ }^{2}$ Sean Kim, ${ }^{2}$ Mark Gregory, ${ }^{2}$ Patrick Danaher, ${ }^{2}$ Joseph Beechem. ${ }^{1}$ Fred Hutchinson Cancer Research Center, Seattle, WA, USA; ${ }^{2}$ Nanostring Technologies, Seattle, WA, USA

Background Although cancer immunotherapies can effectively restore $\mathrm{T}$ cell-mediated immunity leading to sustained clinical responses, these responses are unpredictable partly due to highly heterogeneous phenotypes of tumor-infiltrating lymphocytes (TILs) between patients. Thus, understanding such TILs and their roles in the context of tumor microenvironments (TME) may lead to developing better immunotherapy solutions. The spatial molecular imager (SMI) is a novel spatial transcriptomics platform that allows spatially resolved highdimensional cellular phenotyping for comprehensive TIL profiling. SMI uses fluorescent molecular barcodes to enable in-situ measurement of biological targets on an intact tissue sample. Here, we characterize comprehensive TIL phenotypes and visualize landscape of TILs directly on intact formalinfixed paraffin-embedded (FFPE) tissues using a 1000+-plex RNA panel.

Methods To build multi-omics TIL profiling data sets for renal cell carcinoma (RCC) tissues, we employed scRNA-seq, mass cytometry (CyTOF) and SMI. Peripheral blood mononuclear cells and dissociated cells from matched RCC tumor and adjacent normal tissues were analyzed by CyTOF and single-cell sequencing. Then, SMI profiling of matching FFPE tissues was used to visualize TILs in the context of the TME and to understand relationships between high-dimensional cellular heterogeneity and the spatial organization of cells within a tumor tissue.

Results CyTOF and scRNA-seq analysis of dissociated cells was used to determine the gene expression profiles of numerous cellular subsets. TCR sequencing was also used to assess the extent of clonal expansion and clonotypic relationships between blood and tumor. Consistent with our previous reports, $\mathrm{T}$ cell populations could be segregated based on markers associated with chronic $\mathrm{T}$ cell receptor signaling and many $\mathrm{T}$ cells with an exhausted phenotype were clonally expanded in the tumor but not the blood. In contrast, $\mathrm{T}$ cell clonotypes with bystander phenotypes in the tumor were readily detected as expanded clones in the blood, supporting notion that not all tumor-infiltrating $\mathrm{T}$ cells are specific for tumor antigens. SMI analysis of matched tumor tissue was used to accurately quantify the densities and to determine the spatial organization of all $\mathrm{T}$ cell subsets. In addition, computational methods were used to describe distinct cellular niches within tumors with accurately defined cellular compositions.

Conclusions High dimensional cellular profiling highlights the abundance of bystander $\mathrm{T}$ cell infiltration of RCC tumors. Comprehensive spatial profiling by SMI provides spatial context to the highly diverse immune cell composition of tumor infiltrates.

Ethics Approval Fully anonymous human material was obtained from Northwest Biotrust and given IRB designation of non-human subjects research.

http://dx.doi.org/10.1136/jitc-2021-SITC2021.050 